This is the author's version of the work. It is posted here by permission of the AAAS for personal use, not for redistribution. The definitive version was published in Science 319, p 726. Feb. 8, 2008, DOI:

$10.1126 /$ science. 1151411

http://www.sciencemag.org/cgi/content/full/319/5864/776?ijkey=jlXC/xH6hwYS2\&keytype=ref\&siteid=sci

\title{
Experienced saxophonists learn to tune their vocal tracts
}

\author{
Jer Ming Chen ${ }^{1}$, John Smith ${ }^{1}$ and Joe Wolfe ${ }^{1^{*}}$
}

Science, (2008) 319: p 726

How much do the acoustics of the vocal tract influence performance on single reed instruments (clarinets and saxophones)? To summarize a twenty-five year debate, scientists' opinions have ranged from 'negligible' (1) to 'vocal tract resonant frequencies must match the frequency of the required notes' (2). Musicians' opinions are also diverse. The longevity of the debate is due to the difficulty of making non-perturbing, precise, acoustical measurements inside the mouth during playing (3): i.e. in a variable, humid environment with very high sound levels.

The notes played on wind instruments depend on the acoustic impedance $Z$ : the ratio of sound pressure to the oscillatory component of air flow. Reed instruments usually play at frequencies very near a high, sharp peak in the impedance spectrum of the bore of the instrument. In a standard, simple model (4), the impedance of the vocal tract $Z_{t}$ is in series with that of the bore $Z_{b}$. For most playing conditions $Z_{\mathrm{b}} \gg Z_{\mathrm{t}}$ (e.g. Fig $1 \mathrm{~A}$ ), so acousticians usually neglect the role of the tract.

In saxophones and clarinets, a single reed vibrates to modulate the flow into the instrument, and is in turn driven to vibrate by standing waves in the bore of the instrument. We studied the tenor saxophone, whose mouthpiece is large enough to support devices we constructed for measuring $Z_{\mathrm{t}}$ with minimal perturbation to the player. Five professional and three amateur saxophonists sustained notes for several seconds while we measured $Z_{\mathrm{t}}$ using an acoustic signal comprising 224 sine waves (5, 6 and supporting material).

In Fig 1, (A) and (B) show examples. The dark line is $Z_{\mathrm{t}}$. Upon this are superimposed narrow spikes, which are harmonics of the note being played. The pale line shows $Z_{\mathrm{b}}$ for that note.

Over the lower range of the instrument, the peaks in $Z_{\mathrm{b}}$ are much greater than those in $Z_{\mathrm{t}}$. In this range, the peaks in $Z_{\mathrm{t}}$ varied greatly among players, and showed no consistent relation to the note played (Fig 1A and 1C). In the high ("altissimo") range, however, the professional players consistently tuned a strong peak in $Z_{\mathrm{t}}$ near to or slightly above the fundamental of the note played (Fig 1C).

The amateur players, who did not tune a strong peak in $Z_{\mathrm{t}}$, were unable to play in the altissimo range. A configuration of keys pressed (a fingering) produces a $Z_{\mathrm{b}}$ with several peaks, whose magnitudes decrease at high frequency. In the normal range, the peak of $Z_{\mathrm{b}}$ at the appropriate frequency is large and dominates the series combination $\left(Z_{\mathrm{b}}+Z_{\mathrm{t}}\right)$. In the high range, the relevant peak in $Z_{b}$ is weak (Figs $1 \mathrm{~A}$ and $1 \mathrm{~B}$ are representative). Here, experienced players use a peak in $Z_{\mathrm{t}}$, which may be several times greater than that in $Z_{\mathrm{b}}$, to choose the playing frequency. For instance, 
experienced players can hold a single fingering and play a different note at each of the first dozen peaks in $Z_{\mathrm{b}}$ by tuning $Z_{\mathrm{t}}$

We conclude that the vocal tract resonances have only modest effects on the sounding pitch over much of the instrument's range. However, to play notes in the altissimo range, players learn to tune a resonance of the tract near to the note to be played. Although demonstrated only for saxophone, similar effects are likely to be important in other single and double reed instruments, whose players also report the importance of the tract for special effects, including high register playing.

\section{References and Notes}

1. J. Backus, J. Acoust. Soc. America 78, 17 (1985).

2. P.G. Clinch, G.J. Troup, L. Harris, Acustica 50, 280 (1982).

3. C. Fritz, J. Wolfe, J. Acoust. Soc. America 118, 3306 (2005).

4. A. Benade, in Vocal Fold Physiology, Biomechanics, Acoustics, and Phonatory Control (ed. I.R. Titze, R.C. Scherer), Denver Centre for the Performing Arts, 425 (1985).

5. J. Wolfe, J. Smith, J. Tann, N.H. Fletcher, J. Sound and Vibration, 243, 127 (2001).

6. P. Dickens, J. Smith, J. Wolfe, J. Acoust. Soc. America,121, 1471 (2007).

We thank the Australian Research Council for support, the Yamaha Corporation (Iwata, Japan) for the saxophone and our subjects.

${ }^{1}$ School of Physics, University of New South Wales, Sydney 2052 Australia

*To whom correspondence should be addressed. E-mail: J.Wolfe@unsw.edu.au
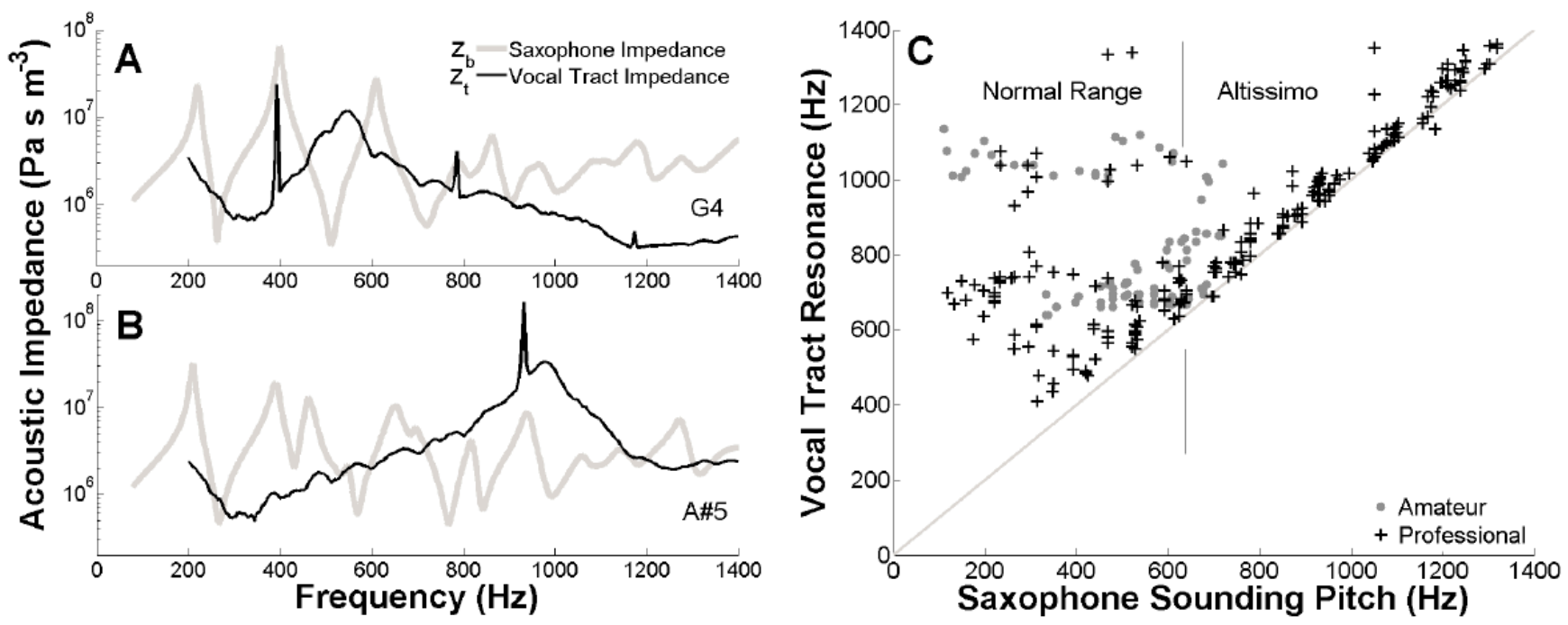

Fig. 1. Representative examples of acoustic impedances of the tract $\left(Z_{t}\right)$ for a professional player $(A)$ for the note G4 (near $400 \mathrm{~Hz}$, in the normal range) and (B) for the note $A \# 5$ (near $940 \mathrm{~Hz}$, altissimo range) (sound files in supporting material). The narrow peaks are harmonics of the notes played, the broad peaks are tract resonances. $Z_{b}$ for the saxophone for each note is shown with a pale line. In $(C)$, the frequencies of the relevant resonance in the vocal tract are plotted against the frequency of the note played. A line indicates the equation tract resonance frequency equals pitch frequency. 


\section{Supporting material (published by Science on line)}

\section{Materials and Methods}

Two different methods were used to measure $Z_{\mathrm{t}}$ (see (1) for a review of methods). For all notes except those in the lowest register of the saxophone, a capillary method (adapted from $(2,3)$ was used, as shown in Fig 2. The signal was an acoustic current comprising 224 frequencies between 200 and $1400 \mathrm{~Hz}$, spaced at $5.383 \mathrm{~Hz}$. The impedance head was calibrated using an acoustically infinite waveguide.

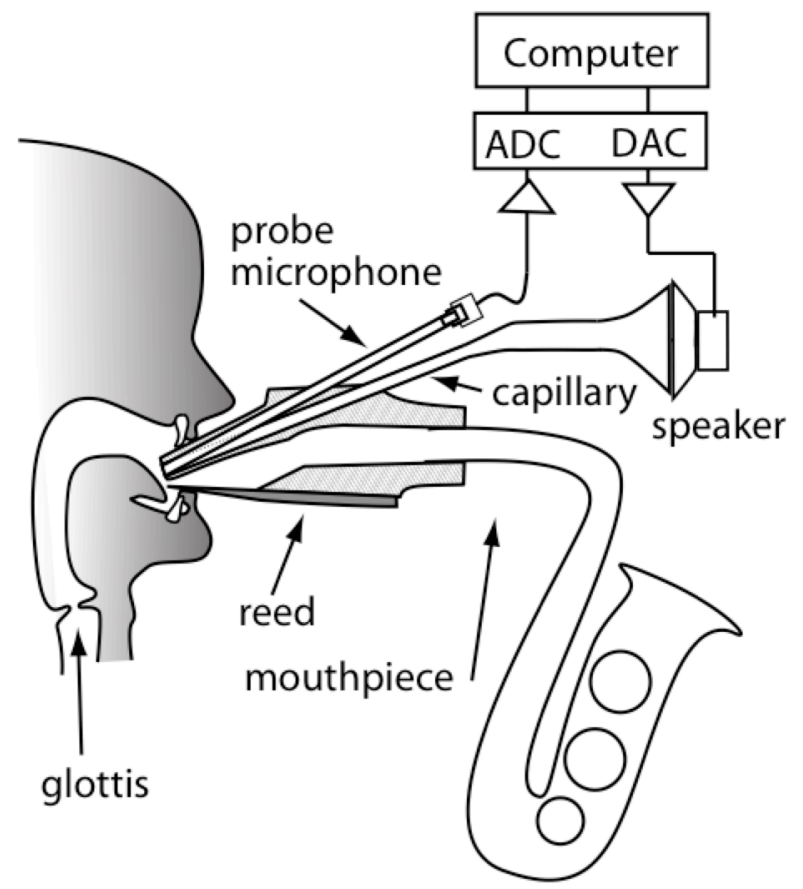

Fig. S1. An acoustic current source injects a sound comprising a broad range of frequencies directly into the vocal tract of the player (not to scale). A probe microphone (Brüel \& Kjaer 4944A) measures the response of the tract to this acoustic current, and also the harmonics of the note being played. The capillary, which is the output of the current source, and the probe of the microphone are both laid in a channel cut into the top of the mouthpiece. (For the multiple microphone method, the waveguide is likewise set into the top of a mouthpiece.) Thus the capillary and probe (or the waveguide) are always acoustically connected to the mouth and are never occluded by the reed, even when the reed completely closes the mouthpiece (called the 'beating reed' condition).

For low notes (in the saxophone's first register), the flow of air in the mouth produced a broad band signal that reduced the signal:noise ratio of measurements, so the multiple microphone method (1) was used. This method was also used to measure the impedance spectra $Z_{\mathrm{b}}$ of the saxophone.

The measurement head for the multiple microphone method had an acoustical impedance of 6 MPa.s.m ${ }^{-3}$. While this was large compared to the peaks in $Z_{\mathrm{t}}$ for low notes, it was not large compared to those in $Z_{\mathrm{t}}$ for high notes. Although the impedance of the head itself does not perturb the measurements, a low value does perturb the players for high notes, because it is in parallel with $\mathrm{Z}_{\mathrm{t}}$. Consequently, the capillary method was used for the medium and high range of the saxophone (second register and above). 
The strong peaks in $Z_{\mathrm{t}}$ that we observe in professional players are consistent with a small glottal opening, although this was not confirmed because players are generally unaware of the state of the glottis. A small glottal opening was observed in very experienced wind players by Mukai (4) using laryngoscopy.

Players of the didjeridu often produce strong peaks in $Z_{t}$ in the range 1-2 kHz, where they may exceed $\mathrm{Z}_{\mathrm{b}}$ for high frequencies. Consequently, they strongly modify the amplitude of some of the high harmonics and thus the timbre (3). That the vocal tract also has (less spectacular) effects on the timbre of other wind instruments is known to both players and some composers (e.g. 5). However, we know of no previous study that shows a strong dependence of the note played on the vocal tract configuration.

1. P. Dickens, J. Smith, J. Wolfe, J. Acoust. Soc. America,121, 1471 (2007).

2. J. Wolfe, J. Smith, J. Tann, N.H. Fletcher, J. Sound and Vibration, 243, 127 (2001).

3. A. Tarnopolsky, N. Fletcher, L. Hollenberg, B. Lange, J. Smith, J. Wolfe, J. J. Acoust. Soc. America 119, 1194 (2006).

4. M. S. Mukai, in Proc. International Symposium on Musical Acoustics, Tokyo, Japan, pp. 239 (1992).

5. Berio, L. "Sequenza V; Solo Trombone" Universal, NY (1966).

\section{Sound files}

A professional plays G4 and A\#5 (written as A5 and C7 for the tenor saxophone). These are the notes used for Figs $1 \mathrm{~A}$ and $1 \mathrm{~B}$ in the paper.

[profG4Ax5.wav] [profG4Ax5.mp3]

An amateur plays G4 correctly. He then plays with the fingering for A\#5. Because he does not tune the vocal tract resonance to A\#5, the instrument plays at its strongest impedance peak, near $200 \mathrm{~Hz}$, which is about G\#3.

[amG4Ax5.wav] [amG4Ax5.mp3]

A professional plays comfortably over the normal range (below about F5) and altissimo range of the tenor saxophone. The two passages cover the range D\#3 to A\#5 (written F4 to C7) and F3 to G5 (written G4 and A6).

[profsample.wav] [profsample.mp3]

An amateur begins a scale at A\#4 (written C6) near the top of the normal range. From F5 (written G6), he fingers the appropriate keys. However, without appropriate vocal tract tuning, the instrument plays at one of the lower impedance peaks.

[amscale.wav] [amscale.mp3] 


\section{One sentence abstract.}

To play the high range of the saxophone, players must learn to tune a strong resonance of their vocal tract to the desired note.

\section{0 word abstract.}

Acousticians have long debated whether and how the resonances of the vocal tract are involved in the playing of clarinet and saxophone. We measured the resonances of saxophonists' vocal tracts directly, while they played. Over most of the instrument's range, there is no simple relation between tract resonances and the note played, and the tract resonances varied among players. In the high (altissimo) range, a strong resonance of the tracts of professional saxophonists was systematically tuned slightly above the desired note. Amateurs, who did not tune a strong resonance, were unable to play notes in the altissimo range. 